\title{
ESTABLECIMIENTO DE UNA COLONIA DE LUTZOMYIA WALKERI (NEWSTEAD, 1914) (DIPTERA: PHLEBOTOMINAE)
}

\author{
ALBERTO MORALES, * CRISTINA FERRO DE CARRASQUILLA, * CRISTINA ISAZA DE RODRIGUEZ. *
}

\begin{abstract}
Con el propósito de investigar la posible implicación de $L u$. walkeri en la transmisión de Leishmania braziliensis panamensis en un foco de leishmaniasis en la vereda EI Mercado del municipio de Mariquita, departamento del Tolima, Colombia, S.A., se estableció una colonia de esta especie de Lutzomyia. Actualmente esta colonia se encuentra en la octava generación, la cual se inició con 301 hembras recolectadas con cebo humano en el año de 1982; el promedio de huevos depositados por cada hembra fue de 29 y el máximo de huevos puestos por una hembra fue de 79. En un ensayo controlado se separaron 1.283 hembras las cuales depositaron 37.118 huevos de los cuales emergieron 9.030 adultos lo que da un rendimiento de $24,3 \%$. EI ciclo evolutivo de huevo a adulto tuvo una duración promedio de 67 días a una temperatura promedio de $21,5^{\circ} \mathrm{C}$ para los adultos y de $23^{\circ}$, para las larvas. Se observó que Lutzomyia walkeri es antropofílico y zoofílico.
\end{abstract}

\section{INTRODUCCION}

Desde febrero de 1979 el Instituto Nacional de Salud de Colombia ha venido realizando estudios epidemiológicos en un foco de Leishmania braziliensis panamensis en algunas veredas del municipio de Mariquita en el departamento del Tolima. (1) Una parte importante del estudio lo ha constituido la investigación de las especies de Lutzomyia que se presentan en el área, la bionomía de las especies halladas, su posible implicación como vectores eficientes de Leishmania braziliensis panamensis y los mecanismos de infección y transmisión del insecto con este parásito.

En desarrollo de este último punto se han tratado de establecer colonias de algunas de las especies de Lutzomyia del foco de Mariquita y el objetivo de esta publicación es el de comunicar el establecimiento de una colonia de Lutzomyia walkeri y dar algunos detalles de su bionomía.
Lu.walkeri fue descrito originalmente por Newstead en $1914^{(2)}$ desconociéndose con exactitud la localidad tipo, pero se sabe que está situada en el río Abuña, en la frontera entre Bolivia y Brazil. (3) De acuerdo con Young, ${ }^{(4)}$ esta especie ha sido encontrada en Panamá, Ecuador, Perú, Brazil y Trinidad. En Colombia, el primer registro de esta especie se hizo en captura realizada durante la noche, en un gallinero, en el municipio de San Vicente de Chucurí, Santander, el 16 de agosto de 1944, recolección hecha por Gast, Osorno y Mangabeira; este material fue estudiado por Sherlock ${ }^{(5)}$ quien describió con él una nueva especie Lutzomyia gasti pero más tarde Young (4) propuso que gasti era conespecífica con walkeri relegando de esta manera a gasti a la sinonimia. Además de San Vicente de Chucurí, esta especie ha sido hallada en Colombia en regiones de Anorí (Antioquia), Solano y Tres Esquinas (Caquetá), Acacías y Puerto Lleras (Meta), Landázuri (Santander), San José del Guaviare (Guaviare) y Mariquita (Tolima).

* Grupo de Entomología - Instituto Nacional de Salud. Apartado 80334, Bogotá. 
Lutzomyia walkeri pertenece al grupo Migonei de Theodor, $1965^{(6)}$ del cual se han encontrado en Colombia cuatro especies.

El establecimiento de colonias de especies de Lutzomyia reviste una gran importancia pues ello permite hacer observaciones de laboratorio sobre el ciclo de vida de la especie colonizada, su fisiología y competencia como vectora de especies de Leishmania y arbovirus, investigaciones acerca de mecanismos de infección y transmisión, realizar estudios genéticos y probar la efectividad de diversos insecticidas. Además, ofrece la oportunidad de ensayar nuevos métodos de mantenimiento de estos insectos en colonias. Sin embargo, de aproximadamente seiscientas especies conocidas de flebotomíneos, menos de veinte han sido colonizadas en gran número por más de diez generaciones ${ }^{(7,8)}$.

Son muchas las razones por las cuales tan poco número de especies han sido exitosamente colonizadas pero ello se debe especialmente a que es difícil el establecimiento y mantenimiento de estas colonias y a que es necesario conocer previamente los hábitos de picadura de la especie y sus requerimientos de humedad, temperatura, alimentación de las larvas, etc.

\section{MATERIAL Y METODOS}

Captura de adultos. La recolección de los adultos se hizo en el corredor de una casa de habitación en la finca El Guayacán, vereda El Mercado, municipio de Mariquita, departamento del Tolima, Colombia S.A., en captura nocturna de las 18:30 a las $21: 30$, con cebo humano y por dos hombres en los días 28 de agosto de 1982; 24, 27 y 28 de septiembre de 1982 , octubre 13 y octubre 20 de 1982; y noviembre 2 de 1982. Las hembras que llegaban a picar o que se encontraban reposando en las paredes exteriores de la casa de habitación eran capturadas con aspirador manual de boca e inmediatamente introducidas en tarros de bambú; terminada la captura, los insectos eran llevados al laboratorio de campo, situado en la misma vereda y a poca distancia del sitio de captura. En el laboratorio de campo los insectos eran soltados dentro de jaulas de tela en cuyo interior se colocaba, sobre una pequeña caja de Petri, un poco de algodón humedecido con solución azucarada, aproximadamente al $30 \%$ en agua. Las jaulas que contenían los adultos se mantenían dentro de un rancho construído con material de guadua y techo de paja; estas jaulas se colocaron sobre soportes de ladrillo los que a su vez se pusieron dentro de bandejas esmaltadas que contenían agua para proteger los adultos de las hormigas.

Dentro del rancho en donde estaban las jaulas, la iluminación era muy pobre de manera que aún durante el día el interior se mantenía en penumbra. La temperatura promedio ambiental del cuarto en donde se mantuvieron las jaulas con los adultos fue de $21,5^{\circ} \mathrm{C}$ y la humedad de $92,11 \%$.

En la mañana del día siguiente a su captura, a las hembras se les permitió alimentarse de un hámster anestesiado con pentotal sódico; el hámster se dejaba dentro de la jaula hasta cuando daba muestras de estar despertando de la anestesia, aproximadamente una hora. A las hembras que no se alimentaban ese día, se les daba oportunidad de tomar sangre en los días subsiguientes.

Las hembras que se llenaban de sangre eran separadas individualmente, en viales de vidrio de $2 / \mathrm{cm}$. de diámetro por $5,5 \mathrm{~cm}$ de profundidad; estos viales eran tapados con un pedazo de tela de muselina la cual se aseguraba con una banda de caucho. Antes de pasar la hembra llena al vial, se colocaba en el fondo un rodete de papel de filtro y sobre éste otro pedazo de papel de filtro recortado en forma de triángulo y al que se le había hecho un agujero en el centro con el fin de que el insecto pudiera circular de un lado al otro del triángulo de papel.

Para mantener la humedad dentro del vial al rodete de papel de filtro del fondo se le agregaban, cada vez que era necesario y con jeringa, unas gotas de agua destilada. En general, la técnica usada para el desarrollo de los insectos fue descrita por KillickKendrick y col. ${ }^{(9)}$

A los viales que contenía las hembras llenas de sangre se les colocaba, sobre la 
tela que cerraba el vial, un algodón humedecido con una solución de azúcar en agua destilada al 30\% todos los días.

Diariamente se examinaban los viales que contenían las hembras y cuando se observaba postura de huevos, se procedía a transferirlos a vasos plásticos preparados para tal propósito; las hembras depositaban la mayoría de sus huevos en el rodete de papel de filtro húmedo que se había colocado en el fondo del vial y sólo unos pocos en sus paredes.

Las capturas de hembras que se hicieron en el campo estaban compuestas por varias especies, de manera que se esperaba hasta que hicieran las posturas de los huevos y entonces sí se procedía a identificarlas; de esta forma fue posible reunir los huevos de una sola especie.

Los vasos plásticos a donde se transferían los huevos tienen la forma de un cono recortado de diámetro superior de $7 \mathrm{~cm}$. e inferior de $5 \mathrm{~cm}$; a estos vasos se les colocó interiormente en el fondo, una capa de yeso de 1,5 a $2,0 \mathrm{~cm}$ de altura. Los huevos se pasaban al vaso plástico con la ayuda de un pincel de pelo de camello y se depositaban directamente sobre la superficie del yeso; en cada vaso plástico se colocaban los huevos de diez hembras, debidamente contados.

Para mantener la humedad de los huevos, al vaso plástico se le perforaba un pequeño agujero, exteriormente, en el centro de la base y se colocaba dentro de una bandeja esmaltada con tapa, en cuyo fondo se ponía gasa apenas húmeda.

Los huevos se examinaron todos los días y un poco antes de que emergieran las primeras larvas se agregaba a los vasos comida en poca cantidad, la que se aumentaba a medida que emergían las larvas e iban desarrollándose a estadios más avanzados.

Los estadios inmaduros se mantuvieron a una temperatura ambiente de $23^{\circ} \mathrm{C}$, en promedio.

La comida para las larvas consta de galleta para ratones y materia fecal de conejo envejecida, mezclada en proporciones iguales. Para preparar la comida, se desecan las deyecciones de conejo, se trituran por separado la galleta y la materia fecal de conejo, se mezclan, se les agrega un poco de agua y se dejan en un desecador a temperatura ambiente por lo menos dos meses. Los hongos crecen y finalmente desaparecen ${ }^{(10)}$. Cuando esto sucede y el olor es similar al de pan integral, está lista para ser utilizada.

Posteriormente aparecieron las pupas, y finalmente, los adultos los cuales se iban pasando del vaso de cría a jaulas de tela. Durante la noche del mismo día en que emergieron los adultos o al siguiente día, se les alimentó colocándoles dentro de la jaula un hámster anestesiado con pentotal sódico, al cual previamente se le había rasurado el abdomen; una vez alimentadas, las hembras se dejaron en la jaula por un día más para que tuvieran mayor oportunidad de realizar la cópula, después de lo cual las hembras se pasaron a los viales individuales para que realizaran la postura.

Inicialmente no se hizo nigún intento para separar las diferentes especies recolectadas en el campo pues se pensó que era preferible hacerlo en una generación posterior, identificando las hembras que hubieran hecho las posturas en viales individuales y reunir los huevos de hembras de una sola especie. En esta forma los huevos de Lutzomyia walkeri se separaron de los de otras especies capturadas y se inició la colonia. De esta manera se logró establecer que la composición de hembras por especie inicial de acuerdo con su porcentaje, era el siguiente: Lu.shannoni $1,2 \%$, Lu.trapidoi $5,1 \%, \mathrm{Lu}$. bifoliata o lichyi $5,9 \%$, Lu.gomezi $13,4 \%$ y Lu.walkeri $74,1 \%$. Los machos que fueron capturados no se tuvieron en cuenta para el anterior cálculo.

\section{RESULTADOS Y DISCUSION}

En las diferentes capturas que se hicieron se recolectaron un total de 406 hembras de las cuales 301 eran Lu.walkeriy con éstas se inició la colonia que ahora está en la octava generación. 
El período de incubación de los huevos es el tiempo que transcurre entre la postura de los huevos y el momento en que emergen las larvas. Los resultados se muestran a continuación:

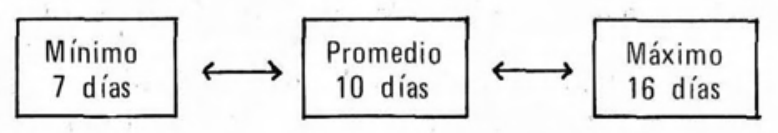

Para tratar de obtener alguna información acerca del ciclo evolutivo de Lu.walkeri, se hizo un seguimiento a 1.283 hembras de la colonia, de diferentes generaciones. Los huevos puestos por cada hembra en los viales individuales fueron contados y depositados en vasos plásticos que tenían yeso en el fondo; en cada vaso se colocaron los huevos correspondientes a 10 hembras, obteniéndose los siguientes resultados:

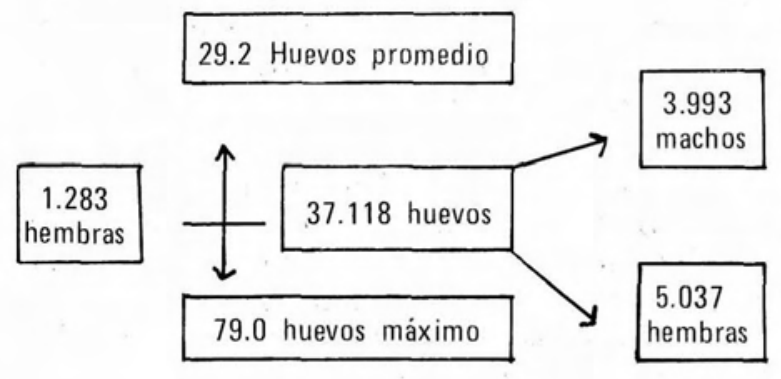

El ciclo evolutivo de huevo a adulto tuvo una duración en días como se ilustra enseguida:

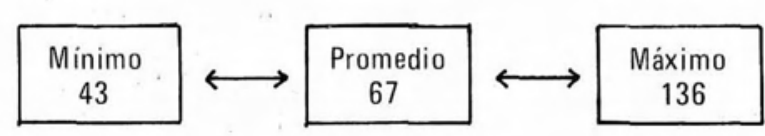

En la colonia las hembras se alimentan muy bien en los hamsters anestesiados que se usan como fuente de suministro de sangre, alimentándose incluso después de unas pocas horas de haber emergido de la pupa. Hemos podido comprobar que Lutzomyia walkeri tiene además hábitos antropofílicos, puesto que las capturas de las hembras con las cuales se inició la colonia se hicieron utilizando cebo humano y, cuando a las hembras de la colonia se les permite alimentarse en humano, lo hacen rápidamente. Adicionalmente, se ha observado que se alimentan muy bien sobre aves (gallinas y pollos). De acuerdo con Young(4), esta sería la segunda especie del grupo Migonei de la cual se sabe que tiene hábitos antropofílicos.

Se observó que las larvas de Lu.walkeri se alimentan en la superficie del substrato.

\section{SUMMARY}

A colony of Lutzomyia walkeri was established in order to investigate its possible role in the transmission of Leishmania braziliensis panamensis in a focus of leishmaniasis in the rural hamlet. El Mercado, township of Mariquita of departament of Tolima, Colombia, S.A. This colony is presently in its 8th generation, which began with 301 females collected with human bait in 1982. The average eggs laid by a female was/29. w ith a maximum of 79 . In a controlled experiment 1283 female were separated, which laid 37.118 eggs out of which 9030 adults were hatched, i.e.an efficiency of $24.3 \%$. The development cycle from egg to adult lasted an average of 67 days at an average temperature of $21.5^{\circ} \mathrm{C}$ for adults and $23^{\circ} \mathrm{C}$ for larvae. Lutzomyia walkeri was seen to be both anthropophilic and zoophilic.

\section{AGRADECIMIENTOS}

A los señores Efrén Téllez y Alvaro Ricaurte, ayudantes del Grupo de Entomología del INS, por su colaboración en el mantenimiento de la colonia de Lu.walkeri.

\section{BIBLIOGRAFIA}

1. Morales A, et al. Aislamiento de tres cepas de Leishmania a partir de Lutzomyia trapidoi en Colombia. Biomédica. 1981; 4: 198.

2. Newstead R. Notes on Phlebotomus, with descriptions of new species. Part II. Bull. Ent. Res. $1.914 ; 5: 179$.

3. Lewis D. J. Redescriptions of two South American sandflies (Diptera : Psychodidae). Proc. Royal. Ent. Soc. London. 1967; 36: 131. 
4. Young G.D. A review of the bloodsucking psychodid flies of Colombia (Diptera : Phlebotominae and Sycoracinae). Agricultural Experiment Stations. Institute of Food and Agricultural Sciences. University of Florida. Gainesville F.A. 1979. Bulletin 806: $266 \mathrm{pp}$.

5. Sherlock I. A. Sobre alguns Phlebotomus e Brumptomyia da Colombia (Diptera, Psychodidae) Men. Inst. Oswaldo Cruz. 1962; 60: 321.

6. Theodor 0 . On the clasification of American Phlebotominae. J. Med. Ent. 1965; 2: 171.

7. Endris R.G, et al. Techniques for laboratory rearing of sand flies (Diptera : Psychodidae). Mosquito News. 1982; 42: 400.
8. Beach R, Young D.G, Mutinga M.J. New phlebotomine sand fly colonies: rearing Phlebotomus martini, Sergentomyia schwetzi, and Sergentomyia africana (Diptera : Psychodidae). J. Med. Entomol. 1983; 20: 579.

9. Killick-Kendrick R., Leaney A.J. and Ready P.D. The Establishment, maintenance and productivity of a laboratory colony of Lutzomyia longipalpis (Diptera : Psychodidae). J. Med. Ent. 1977; 1 3: 429- 440 .

10. Young D.G, Perkins P. V, Erdris R.G, A larval diet for rearing phlebotomine sand flies (Diptera : Psychodidae). J. Med. Entomol. 1981; 18: 446. 


\section{INSTRUCCIONES PARA LOS AUTORES}

1. Biomédica la revista del Instituto Nacional de Salud recibirá para publicación únicamente artículos originales e inéditos.

2. La revista aceptará artículos que contribuyan a ampliar los conocimientos sobre biomedicina realizadas, tanto en el Instituto Nacional de Salud como en cualquier otro centro investigativo.

Dichos artículos deberán llenar los siguientes requisitos:

a) Ser enviados al editor de la revista, Apartados 80334 y 80080, Zona 6, Bogotá, D.E., Colombia S.A.

b) Ser escritos a máquina, a doble espacio, en original y una copia, dejando márgenes de 4 cms. a la izquierda y $2 \mathrm{cms}$. a la derecha. El original en papel blanco, grueso, tamaño carta.

c) Ser escritos en español con resúmenes en español e inglés.

d) Tener un título conciso. Podrán tener, si fuere necesario, un subtítulo explicativo.

e) Llevar los nombres del autor o los autores inmediatamente después, indicando con asteriscos, en el pié de página, su título académico y la institución en la cual se realizó el trabajo.

f) Incluir en el texto del trabajo: Introducción, Materiales y Métodos, Resultados, Discusión, Conclusiones y Referencias Bibliográficas.

g) Las citas bibliográficas se harán en el texto en forma consecutiva, utilizando números arábigos y deberán aparecer, en el mismo orden numérico de citación. La referencia se presenta así: apellido del autor, seguido de las iniciales de su nombre, título del artículo, nombre abreviado de la revista, año de publicación, volumen, número y pagina .

Ejemplo: Barrow CH. Criptococcosis in animals. J.A. M.A. 1955, 127: 125.

Para la citación de libros se seguirá un orden similar, así: Pearse A., Texbook of Biochemistry. Saunders Edt., 1979; pp 49-50.

h) Los cuadros, gráficas y figuras deben numerarse en forma consecutiva con números arábigos y ser presentados en papel fotográfico brillante, en blanco y negro, manteniendo individualmente una proporción de 2 × 3 . Dicho material debe ser de calidad y presentación impecables. En hoja aparte se incluirá la leyenda respectiva.

3. La revista también aceptará para publicación: actualizaciones, memorando, revisiones, comunicaciones breves, cartas al editor, revisión de resúmenes e informes técnicos.

4. Todo material propuesto para publicación será revisado por el Comité Editorial. El Editor informará a los autores, tanto sobre la recepción de los trabajos, como sobre la decisión final que se tome.

5. La revista se reservará el derecho de aceptar o rechazar los artículos y podrá hacer sugerencias que tiendan a mejorar su presentación. Para un mejor cumplimiento de esta función el Comité Editorial podrá consultar a especialistas en la materia.

6. Los originales de los artículos publicados permanecerán en los archivos de la revista; aquellos no aceptados para publicación, serán devueltos a sus autores.

7. El autor principal recibirá libre de costo 5 ejemplares de la revista. Los reimpresos deberán ser sufragados por el autor.

NOTA: Las personas interesadas en adquirir la revista podrán hacerlo en la biblioteca del Instituto Nacional de Salud, a un costo de ciento veinticinco pesos m/cte. $(\$ 125.00)$ cada ejemplar, o tomando una suscripción anual. 